\title{
Aims and opportunities in clinical anatomy
}

\author{
R Putz
}

Ludwig-Maximilians-Universität München, Anatomische Anstalt, Pettenkoferstraße 11, D-80336 München, Germany

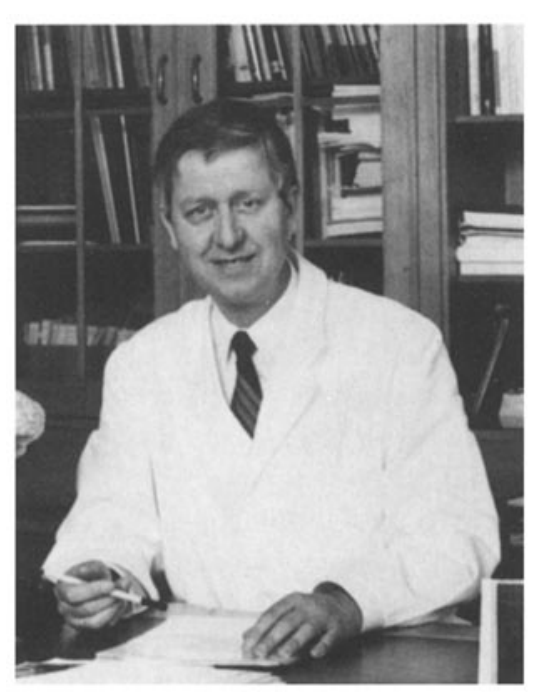

A European Congress provides an ideal opportunity both for analysing the development of our subject critically and for attempting an assessment of its present position.

The subject of anatomy has undergone a dramatic transformation within the space of a few years, and it does seem as if the classical concept of morphology as the foundation of, and key to, medical practice (Vesalius) has been somewhat pushed aside. Specialisation in the field of clinical anatomy with its manifold branches from the dissecting room to the electron microscope has become both impressive and exceedingly complex.

The anatomist, once the direct support of his practising colleagues (hic locus est ubi mors gaudet succurrere vitae), has meanwhile been replaced by clinical standardisation, by the textbook and by the personal initiative of clinicians. The gulf between anatomy and the clinical demands made upon it has grown greater. In many countries (France, for example), the subject has been completely taken over by our clinical colleagues.

\section{What arguments can be brought forward in support of the future viability of our subject?}

\section{The future of clinical anatomy lies in the provision of more "sophisticated" further training}

This argument is based on the fact that anatomic principles underlie practically all everyday clinical activities. Even special clinical situations are adequately presented in the literature, so that the clinician can easily, with a little effort, find his own way. This also includes the communication of known material from the point of view of new insights into its clinical application. The oral and clinical activities of the anatomist also help to facilitate more rapid and direct interaction with more basic information.

The precondition for high quality collaboration is that the anatomist has specialised knowledge of the concrete clinical questions.

\section{Clinical anatomy provides the medical student with basic morphologic understanding}

This approach reduces our specialty almost entirely to that of teaching material, which does seem to be increasingly happening in a number of countries. The anatomist introduces the first-year student to fundamental morphology and to a more or less deductive functional interpretation of the basis of medical practice. So long as this actually involves specimens, and the anatomist is indeed imparting threedimensional concepts, this task is without doubt, as it always was, a key part of medical training. 
The quality of the teaching is therefore certainly dependent upon the teacher's experience and skill, and upon the methodologic training of the student.

\section{Clinical anatomy endeavours to be scientific}

If one seeks to understand only superficially how and by what criteria the significance of each faculty and its subdivisions are to be judged by the scientific community, it is undoubtedly clear that it is by following this third path that there is any possibility of a positive future development. If this is not realised, the scientist must, within his own subject, turn towards other more promising disciplines. Even without any further theoretical discussion, the fate of the subject would thus be sealed.

\section{What, however, is the "scientific concept" of clinical anatomy?}

Answering this question first requires coming to terms with the concept of modern science. If one is prepared to forego the sometimes bitter experiences of the superficially amusing aphorism "science is what scientists recognise as science", it becomes necessary to take a look at the historical development underlying the scientific idea of a "total anatomy".

Anatomy was at first only morphology, and its original objective was always to describe a particular "type". This included the description of single cases, from which it was possible to recognise statistically reliable "normal cases", as well as to assemble a collection of special variants. This was true both for gross structure (macroscopic anatomy) and for microscopic objects (fine structure), as is still the case today. Admittedly one no longer expects, generally speaking, to make any obviously new discoveries, although modern methods can certainly lead to new and surprising findings (CT-MRI, for example). Anatomy concerns itself only secondarily with the understanding and analysis of form, in which "understanding" is to be taken as a word of many meanings, and capable of being reached by many different paths:

a. Understanding form in terms of ontogeny.

b. Understanding form in terms of phylogeny.

c. Understanding form in terms of function and adaptation to changes in function.

d. Understanding form in terms of disease.

Clinical anatomy cannot be classified under any of these headings. It is a blanket term for any way of presenting morphologic results in a form suitable for everyday clinical practice. It is therefore futile to argue about the existence of "more" and "less" important subdivisions of our subject. The common aim and object is its direct usefulness to our clinically active colleagues.

\section{What is the scientific future of clinical anatomy?}

This apparently explicit question can only be given an ambiguous answer. In any case, its scientific impact is not determined by its clinical utility. The scientific nature of a subject is determined directly by the theoretical claims of individual investigations and the methodology employed; it is, however, perfectly free to use any scientific paths available. The question is not therefore "has clinical anatomy a scientific future?", but "is a particular investigation or topical theme itself scientific?". The clinical anatomist must therefore make every effort to achieve direct access to, and contact with, the clinician, and above all to take into account in his work of the theoretical scientific claims which the historical development of our subject has provided.

Clearly formulated theoretical basic principles accepted as indica- tors or as procedural guidelines within a natural science offer a whole range of approaches:

a. Descriptive or empirical methods; the acquisition of new knowledge depends upon either the development of a new process or on the demonstration of morphologic material which has hitherto gone unrecognised, or unrecognised in its present form. The claim to be scientific is based upon the design of the process and the originality of the discovery.

b. Analytic methods; the acquisition of knowledge here depends upon perceiving the relationship of already known morphologic structure with new methods for attaining new ends. The claim to be scientific depends upon the analytic procedures chosen.

c. Comparative methods; the acquisition of knowledge depends upon increasing our understanding of morphologic facts by comparing them with the conditions in certain other species. The claim to be scientific depends upon the ability to derive a new theory about some aspect of evolution.

d. Reductive (biologically experimental) methods; the acquisition of knowledge depends upon asking the right questions, and thus confirming some aspect of a general theory of natural science in the widest sense. The claim to be scientific depends upon formulating a precise hypothesis which can be clearly answered with "yes" or "no" as the result of experiments, and which therefore confirms or destroys a fundamental existing theory.

The way lies open before us and the direction is clear. It is up to us to proceed in the most professional manner possible. The coming together of interested colleagues and the readiness to enter into constructive self-critical discussion are required here. Let us take advantage of this European Congress to promote the development of clinical anatomy as a true science. 